\title{
Rapid learning of rapid temporal contexts
}

\author{
Carly R. Mayberry \\ University of Queensland, Brisbane, Queensland, Australia \\ EVAN J. LIVESEY \\ University of Sydney, Sydney, New South Wales, Australia \\ AND \\ Paul E. Dux \\ University of Queensland, Brisbane, Queensland, Australia
}

\begin{abstract}
In an information-rich visual world and with limited attentional resources, what visual cues allow humans to efficiently navigate their environment? One key environmental characteristic is that stimuli rarely appear in isolation and typically coincide with other specific items that provide cues regarding where and when to guide our attention. Indeed, a predictive spatial context of distractors can enhance the deployment of attention to a target location (Chun \& Jiang, 1998). However, can a predictive, temporal sequence of distractors, which do not enter working memory, cue when to allocate attention? Previous studies addressing this question have employed relatively long $(\leqslant 500 \mathrm{msec} / \mathrm{item})$ stimulus exposure durations. Thus, this temporal cuing may require extensive processing of the distractors. Here, we show that a rapidly presented $(\sim 100 \mathrm{msec} /$ item $)$, predictive, temporal context, where stimuli undergo only preliminary analysis, can facilitate the deployment of attention to a specific temporal location.
\end{abstract}

In everyday life, virtually all visual environments we encounter present a myriad of items of information to analyze. Given our inability to represent all this information consciously, an enduring question is what are the processes involved in isolating the relevant from the irrelevant information (Pashler, 1998). Put differently, how do we select the stimuli that are to undergo extended processing and discard those that are less important for behavior after only limited analysis? A common answer is that, when viewing a scene, we initially adopt a diffuse attentional state (fleeting, capacity unlimited, low resolution), but after identifying items of interest, these become the subject of focused visual attention (sustained, capacity limited, high resolution), allowing them to be consolidated into working memory for report (Chun \& Potter, 1995; Duncan, 1980; Treisman \& Gelade, 1980).

What is the fate of distractor items that are not selected for extended processing? Is the information from these stimuli simply discarded, or is it able to influence behavior? In our everyday environment, the location and identity of both target and distractor stimuli is highly correlated (e.g., if one is looking for a letter box, this normally appears on a street with cars on it, not in the middle of a field surrounded by horses). Thus, distractors are often predictive of targets, and therefore, an efficient biological system should employ this information in order to facilitate processing and overcome its capacity limitations.

The influence of predictive distractors on attention and memory can be demonstrated in the laboratory by em- ploying the contextual-cuing paradigm in visual search (Chun \& Jiang, 1998). Visual search is a popular method for studying how humans spatially distribute attention (Wolfe \& Horowitz, 2004). Here, in a single screen, subjects are presented with a target that is spatially displaced among distractor stimuli and attentional efficiency is assessed by examining the speed with which subjects locate the target as the number of distractors increases. In visual search studies, the distractor configurations are randomly varied from trial to trial. To the subject, a standard spatial contextual-cuing trial appears identical to one from a visual search paradigm; the difference is that, on half the trials, the distractor configuration is predictive of a specific target location/identity. What is typically observed under these conditions is that as the trial number increases, subjects implicitly (Chun \& Jiang, 2003; but see Smyth \& Shanks, 2008) learn these predictive configurations and become faster at directing attention to, and selecting the response for, the target (Kunar, Flusberg, \& Wolfe, 2008).

Spatial contextual cuing has now been demonstrated across a variety of experimental conditions (for a review, see Brady \& Chun, 2007); however, there has been limited research on contextual cuing from predictive information distributed across time. Some information on this issue may be gleaned from paradigms such as the serial reaction time task, where subjects incidentally learn invariant response patterns to sequences of attended items (Nissen \& Bullemer, 1987). Similarly, associative learning studies have shown that a particular target stimulus appearing in

P. E.Dux, paul.e.dux@gmail.com 
a sequential stream of distractors can facilitate an impending motor response (e.g., Livesey, Harris, \& Harris, 2009). In addition, research on statistical learning has shown that subjects can learn associations between targets that appear in specific sequences, which increases subjects' familiarity ratings of these items (Fiser \& Aslin, 2002; TurkBrowne, Jungé, \& Scholl, 2005; Turk-Browne \& Scholl, 2009). Importantly, however, temporal contextual cuing is fundamentally distinct from all these paradigms because (1) it is the predictive information from irrelevant distractor items, which are not selected for extended processing and, therefore, do not enter working memory, rather than that from targets that cues behavior and/or (2) the predictive information guides attention, rather than a motor response that can be primed with limited cognitive processing of the predicted event. Put differently, in temporal contextual cuing, attention is directed to a temporal point in a stream of information where a decision has to be made (i.e., which stimulus was seen); however, the predictive information provides no cue regarding what decision should be made (i.e., the stimulus that was seen).

To date, only one study has examined temporal contextual cuing: Olson and Chun (2001) manipulated sequentially presented distractor identities and event durations, so that they either did or did not predict the temporal position of a target, and found that reaction time was facilitated under predictive conditions. In addition, follow-up tests suggested that subjects were unaware of the repeated distractor streams, suggesting that this learning, like its spatial counterpart, is largely implicit in nature. Similarly, Swallow and Zacks (2008) found that statistical learning of a sequence of arm gestures improved the detection of a predicted target hand gesture even when the sequence predicted only the timing, rather than the identity, of the target gesture.

Although these two results demonstrate that the sequential structure in the visual environment plays an important role in directing attention, in both studies and in many of the paradigms described above, stimuli were presented relatively slowly (e.g., $>500 \mathrm{msec}$, on average; but see Livesey et al., 2009). Thus, at present, the extent to which these predictive distractors must be processed before they enhance performance or the rate at which predictive information can be extracted from a temporal stream of stimuli is unknown. Specifically, at long exposure durations, subjects can easily select all distractor stimuli, have them enter working memory (where they are briefly available to conscious processing), and then inhibit these irrelevant items when they are determined to be nontarget stimuli. Thus, it may be necessary for items to be selected in order for learning to be observed from distractors. Indeed, the results from a statistical-learning study by Turk-Browne et al. (2005) suggest that learning is seen only for selected items or those that are part of a predefined relevant item attentional set. Even in spatial contextual cuing, where exposure duration is unconstrained, results suggest that the predictive configuration of distractors must at least be presented in the attended color before they facilitate target detection (Jiang \& Leung, 2005). Here, we investigated whether nonselected items, which do not enter working memory, can give rise to learning by examining whether temporal contextual cuing is observed under conditions of rapid serial visual presentation (RSVP; $110 \mathrm{msec} / \mathrm{item}$ ). In RSVP, it is known that stimuli undergo only preliminary analysis; the conceptual representations of the distractors are fleetingly activated but are not encoded into short-term memory (Chun \& Potter, 1995; Dux \& Marois, 2009; Potter, 1975, 1976, 1993). To preview our results, we demonstrated temporal contextual cuing under RSVP conditions and found that this learning is rapid in nature and not dependent on temporally salient predictive distractors.

\section{METHOD}

The University of Queensland Ethical Review Board approved the experimental protocol. Thirty-two members of the University of Queensland community participated in the study. Subjects were excluded if they were below $70 \%$ accuracy (chance $=50 \% ; n=4$ ), resulting in 28 subjects being retained in the sample. In the experiment, two variables were examined: distractor stream type (predictive vs. nonpredictive) and trial block (1-8).

RSVP streams contained uppercase letters drawn from the alphabet, excluding I, L, O, and Q. All the stimuli were white, and the background was gray. The target was the letter X or Y, which could appear at Serial Positions 8-17. Thus, each trial contained one target and 20 distractors, meaning that at least 7 distractors preceded the target, whereas at least 4 followed it (no stimuli were repeated in the stream). A fixation square presented for $500 \mathrm{msec}$ appeared at the start of all trials, and each stimulus was presented for $110 \mathrm{msec}$ in the center of the screen. The subjects responded to the presence of the letter $\mathrm{X}$ or $\mathrm{Y}$ as quickly and as accurately as possible by pressing the " $\mathrm{J}$ " or " $\mathrm{K}$ " key, respectively, which were mapped to the index and middle fingers of the right hand (reaction times were accepted for correct trials from 200 to 2,000 msec). Each block contained 40 trials, with the 10 possible target serial positions appearing an equal number of times. Blocks were initiated by the subjects' pressing the space bar, and trials had an interstimulus interval of 2,000 msec. The key manipulation (not communicated to the subjects prior to the experiment) was that, on half the trials in each block, the order of the distractors was randomly generated (nonpredictive trials), whereas on the remaining half, a distinct distractor sequence within the RSVP stream was paired with a target serial position (predictive trials). These sequences were different for each subject and made up of all of the distractor stimuli preceding the target, excluding the first item presented and the item directly before it (see Figure 1A). The first stream item and the distractor immediately preceding the target were randomized, since these are temporally salient stimuli (Olson \& Chun, 2001). Ten unique predictive sequences were used, each appearing twice per block, once with the $\mathrm{X}$ and once with the $\mathrm{Y}$ target. Thus, the distractors predicted only target stream position, not the required motor response. The subjects performed eight blocks of trials with the predictive distractor streams held constant, and, within the blocks, conditions were randomly ordered. At the conclusion of each trial, feedback was given to the subjects on the basis of performance: either "correct" presented in green (RGB: 02550 ) or "incorrect" presented in red (255 00$)$. In addition, at the conclusion of the experimental trials, the subjects completed a follow-up test to assess whether or not they were aware of the distractor stream manipulation. Here, they viewed 120 trials, half of which were predictive, and judged, with no time pressure, whether or not they had seen each before. The large number of follow-up trials were included to increase the accuracy of our consciousness assessment (see Smyth \& Shanks, 2008). The logic behind this test was that if learning had occurred primarily in the absence of awareness, the subjects would be at chance at recognizing the repeated distractor streams. The experiment was programmed in MATLAB using the Psychophysics Toolbox extension (Brainard, 1997; Pelli, 1997) and was presented using a Mac minicomputer and a 21 -in. Dell/Sony CRT monitor. 


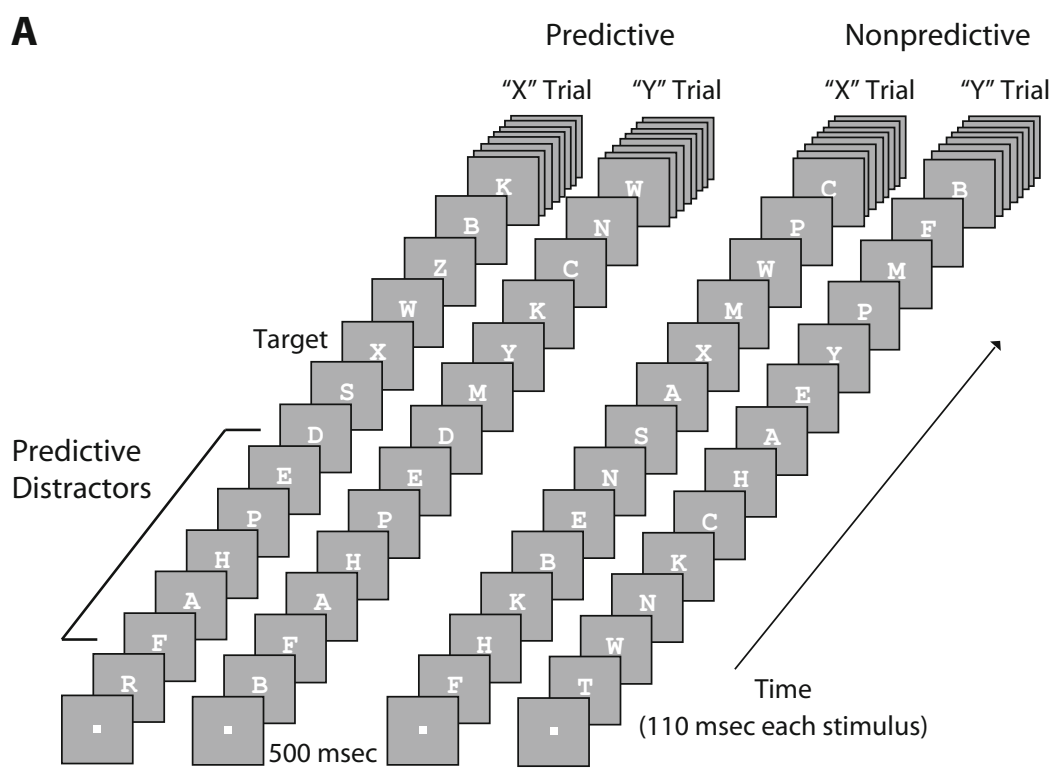

B

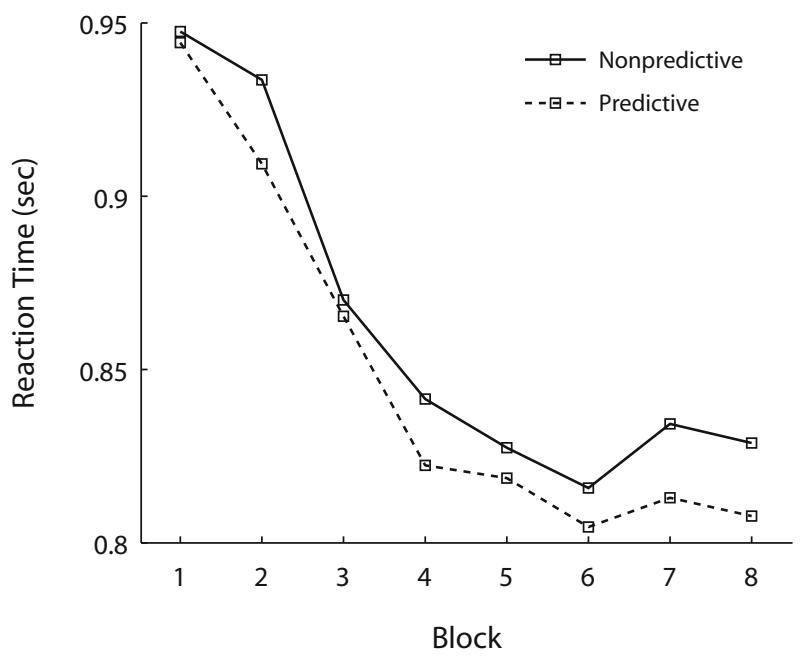

Figure 1. (A) Rapid serial visual presentation task. Subjects searched for the target $X$ or Y. On half the trials, the distractor sequence was predictive of the specific target temporal position (not its identity); on the remaining half, the distractor sequence was randomly generated. (B) Reaction time as a function of trial block and distractor stream type.

\section{RESULTS AND DISCUSSION}

A 2 (predictive vs. nonpredictive) $\times 7$ (Blocks 2-8; Block 1 was considered practice) repeated measures ANOVA demonstrated that the subjects' performance on the task as a whole improved with experience as mean reaction time decreased across block $[F(6,162)=6.6$, $\left.p<.001, \eta_{\mathrm{p}}^{2}=.2\right]$. Importantly, significant learning of the distractor contexts was observed, since the subjects responded more quickly on predictive trials than on nonpredictive trials $\left[F(1,27)=6.5, p<.02, \eta_{\mathrm{p}}^{2}=.2\right.$; see Figure 1B]. However, there was no interaction $(F<1)$. Thus, it appears that learning is rapid under RSVP conditions, with only a few presentations of the predictive streams necessary for temporal contextual cuing to be observed. Indeed, when the first block of trials was examined, no benefit of the predictive distractor contexts was observed $(t<1)$, suggesting that after only two presentations, attentional deployment was facilitated by predictive distractor information (we have now replicated this finding numerous times in our lab, across a variety of stimulus conditions). There was no evidence of learning when the accuracy data were examined $(p>.1)$; however, accuracy improved across blocks $\left[F(6,162)=3.6, p<.05, \eta_{\mathrm{p}}^{2}=\right.$ .07 ; overall accuracy $=87 \%]$. In addition, the subjects were no different from chance during the follow-up test 
$(50.1 \%, t<1)$, suggesting they were not aware of the distractor manipulation.

These results demonstrate that subjects rapidly learn predictive distractor contexts, which helps them in distributing temporal attention. Importantly, it appears that temporal contextual cuing can be observed without the predictive items being selected for working memory, since, at RSVP rates, distractors undergo only preliminary conceptual processing (Chun \& Potter, 1995; Dux \& Marois, 2009; Potter, $1975,1976,1993)$. This extends previous studies in which relatively slow exposure durations were employed (Olson \& Chun, 2001), since, from these experiments, the level of processing necessary for temporal contextual cuing to be observed could not be determined. In addition, the present results must reflect the predictive information's directing attention to a point in time, rather than a specific motor response or familiarity judgment, since, under the present conditions, the distractors predicted only the target's temporal location. The speed with which this learning takes place also accords with recent psychophysiological studies in which statistical learning of regular stimulus sequences was observed after a small number of repetitions (Abla, Katahhira, \& Okanoya, 2008; Turk-Browne, Scholl, Chun, \& Johnson, 2009). Finally, given that we employed RSVP and that the subjects could not explicitly recognize the predictive distractor streams during the follow-up, it appears not only that temporal contextual cuing can be observed after only a limited analysis of the distractors, but also that it is largely implicit in nature. The length of our follow-up test (120 trials) is an important feature of the study, since Smyth and Shanks (2008) have suggested that failures to find evidence of conscious processing in spatial contextual cuing were due to relatively few follow-up samples being taken.

\section{CONCLUSION}

Under conditions of rapid stimulus presentation, where distractor stimuli undergo only preliminary processing, subjects are able to extract predictive information from these stimuli, which helps them deploy temporal attention more effectively. This learning appears to be implicit, occurs rapidly, is influenced by the entire distractor stream preceding the target, and is not reliant on temporally salient items. Thus, it appears that in temporal visual search, a key method for overcoming the severe capacity limits of the human information-processing system is to rely on the environment to cue when attention should be deployed.

\section{AUTHOR NOTE}

Both P.E.D. (DP0986387) and E.J.L. (DP0880865) were supported by Australian Research Council APD fellowships. Correspondence concerning this article should be addressed to P. E. Dux, School of Psychology, University of Queensland, 463 McElwain Building, St. Lucia, QLD 4072, Australia (e-mail: paul.e.dux@gmail.com).

\section{REFERENCES}

Abla, D., Katahhira, K., \& OKanoya, K. (2008). On-line assessment of statistical learning by event-related potentials. Journal of Cognitive Neuroscience, 20, 952-964.
Brady, T. F., \& CHUN, M. M. (2007). Spatial constraints on learning in visual search: Modeling contextual cuing. Journal of Experimental Psychology: Human Perception \& Performance, 33, 798-815.

Brainard, D. H. (1997). The Psychophysics Toolbox. Spatial Vision, 10, 433-436

Chun, M. M., \& Jiang, Y. (1998). Contextual cueing: Implicit learning and memory of visual context guides spatial attention. Cognitive Psychology, 36, 28-71.

CHun, M. M., \& JiAng, Y. (2003). Implicit, long-term spatial contextual memory. Journal of Experimental Psychology: Learning, Memory, \& Cognition, 29, 224-234.

Chun, M. M., \& PotTer, M. C. (1995). A two-stage model for multiple target detection in rapid serial visual presentation. Journal of Experimental Psychology: Human Perception \& Performance, 21, 109-127.

Duncan, J. (1980). The locus of interference in the perception of simultaneous stimuli. Psychological Review, 87, 272-300.

Dux, P. E., \& MaroIs, R. (2009). The attentional blink: A review of data and theory. Attention, Perception, \& Psychophysics, 71, 1683-1700.

FisER, J. Z., \& ASLIN, R. N. (2002). Statistical learning of higher-order temporal structure from visual shape sequences. Journal of Experimental Psychology: Learning, Memory, \& Cognition, 28, 458-467.

JiAng, Y., \& LEUNG, A. W. (2005). Implicit learning of ignored visual context. Psychonomic Bulletin \& Review, 12, 100-106.

Kunar, M. A., Flusberg, S. J., \& Wolfe, J. M. (2008). Time to guide: Evidence for delayed attentional guidance in contextual cueing. Visual Cognition, 16, 804-825.

Livesey, E. J., Harris, I. M., \& Harris, J. A. (2009). Attentional changes during implicit learning: Signal validity protects a target stimulus from the attentional blink. Journal of Experimental Psychology: Learning, Memory, \& Cognition, 35, 408-422.

Nissen, M. J., \& Bullemer, P. (1987). Attentional requirements of learning: Evidence from performance measures. Cognitive Psychology, 19, 1-32.

Olson, I. R., \& Chun, M. M. (2001). Temporal contextual cuing of visual attention. Journal of Experimental Psychology: Learning, Memory, \& Cognition, 27, 1299-1313.

Pashler, H. E. (1998). The psychology of attention. Cambridge, MA: MIT Press.

PeLLI, D. G. (1997). The VideoToolbox software for visual psychophysics: Transforming numbers into movies. Spatial Vision, 10, 437-442.

Potter, M. C. (1975). Meaning in visual search. Science, 187, 965966.

Potter, M. C. (1976). Short-term conceptual memory for pictures. Journal of Experimental Psychology: Human Learning \& Memory, 2, 509-522.

Potter, M. C. (1993). Very short-term conceptual memory. Memory \& Cognition, 21, 156-161.

Smyth, A. C., \& Shanks, D. R. (2008). Awareness in contextual cuing with extended and concurrent explicit tests. Memory \& Cognition, 36, 403-415.

Swallow, K. M., \& Zacks, J. M. (2008). Sequences learned without awareness can orient attention during the perception of human activity. Psychonomic Bulletin \& Review, 15, 116-122.

Treisman, A. M., \& Gelade, G. (1980). A feature-integration theory of attention. Cognitive Psychology, 12, 97-136.

Turk-Browne, N. B., Jungé, J. A., \& Scholl, B. J. (2005). The automaticity of visual statistical learning. Journal of Experimental Psychology: General, 134, 552-564.

Turk-Browne, N. B., \& Scholl, B. J. (2009). Flexible visual statistical learning: Transfer across space and time. Journal of Experimental Psychology: Human Perception \& Performance, 35, 195-202.

TurK-Browne, N. B., Scholl, B. J., Chun, M. M., \& Johnson, M. K. (2009). Neural evidence of statistical learning: Efficient detection of visual regularities without awareness. Journal of Cognitive Neuroscience, 21, 1934-1945.

Wolfe, J. M., \& Horowitz, T. S. (2004). What attributes guide the deployment of visual attention and how do they do it? Nature Reviews Neuroscience, 5, 1-7.

(Manuscript received October 7, 2009; revision accepted for publication December 19, 2009.) 